\title{
General-relativistic instability in hylotropic supermassive stars
}

\author{
L. Haemmerlé
}

\begin{abstract}
Département d'Astronomie, Université de Genève, chemin des Maillettes 51, 1290 Versoix, Switzerland e-mail: lionel.haemmerle@unige.ch
\end{abstract}

Received 2 November 2020 / Accepted 14 November 2020

\begin{abstract}
Context. The formation of supermassive black holes by direct collapse would imply the existence of supermassive stars (SMSs) and their collapse through the general-relativistic (GR) instability into massive black hole seeds. However, the final mass of SMSs is weakly constrained by existing models, in spite of the importance of this value for the consistency of the direct collapse scenario. Aims. We estimate the final masses of spherical SMSs within the whole parameter space that is relevant to these objects.

Methods. We built analytical stellar structures (hylotropes) that mimic existing numerical SMS models, accounting for full stellar evolution with rapid accretion. From these hydrostatic structures, we determine ab initio the conditions for GR instability and compare the results with the predictions for full stellar evolution.

Results. We show that hylotropic models predict the onset of GR instability with a high level of precision. The mass of the convective core appears as a decisive quantity. The lower it is, the larger the total mass required for GR instability. The typical conditions for GR instability feature a total mass of $\gtrsim 10^{5} M_{\odot}$ with a core mass of $\gtrsim 10^{4} M_{\odot}$. If the core mass remains below $10^{4} M_{\odot}$, total masses in excess of $10^{6}-10^{7} M_{\odot}$ can be reached.

Conclusions. Our results confirm that spherical SMSs forming in primordial, atomically cooled haloes collapse at masses below $500000 M_{\odot}$. On the other hand, accretion rates in excess of $1000 M_{\odot} \mathrm{yr}^{-1}$, leading to final stellar masses of $\gtrsim 10^{6} M_{\odot}$, are required for massive black hole formation in metal-rich gas. Thus, the different channels of direct collapse imply distinct final masses for the progenitor of the black hole seed.
\end{abstract}

Key words. stars: massive

\section{Introduction}

Black hole seeds with masses of $\gtrsim 10^{4} M_{\odot}$ that are born via direct collapse during the formation process of their host galaxies appear to be a natural explanation for the existence of the most massive black holes observed at high redshift (e.g. Rees 1978, 1984; Volonteri et al. 2008; Woods et al. 2019; Haemmerlé et al. 2020). The direct progenitors of these objects would be supermassive stars (SMSs) growing by accretion at rates of $\dot{M}>$ $0.1 M_{\odot} \mathrm{yr}^{-1}$ until they collapse through the general-relativistic (GR) instability (Chandrasekhar 1964). SMSs could form at the centre of protogalaxies in the absence of molecular hydrogen (e.g. Haiman et al. 1997; Omukai 2001; Bromm \& Loeb 2003; Dijkstra et al. 2008). Hydrodynamical simulations show that the core of such primordial, atomically cooled haloes would collapse with negligible fragmentation at inflows of $0.1-10 M_{\odot} \mathrm{yr}^{-1}$ below parsec scales (Latif et al. 2013). Another efficient mechanism for the formation of massive black hole seeds is the merger of massive, gas-rich galaxies (Mayer et al. 2010, 2015; Mayer \& Bonoli 2019). In addition, to allow for inflows as high as $10^{5} M_{\odot} \mathrm{yr}^{-1}$ at parsec scales, this formation channel does not require primordial chemical composition and metallicities up to solar are expected. Thus, the progenitors of massive black hole seeds could as well be Population I (Pop I) or Population III (Pop III) SMSs.

The evolution of SMSs under rapid accretion has been addressed in several works over the last decade (Begelman 2010; Schleicher et al. 2013; Hosokawa et al. 2013; Sakurai et al. 2015; Umeda et al. 2016; Woods et al. 2017; Haemmerlé et al. 2018a,b, 2019; Haemmerlé \& Meynet 2019).
For $\dot{M} \gtrsim 0.01 M_{\odot} \mathrm{yr}^{-1}$, they evolve as red supergiant protostars (Hosokawa et al. 2013), with most of their mass contained in a radiative envelope (Begelman 2010). As nearly-Eddington stars, their pressure support and entropy content are dominated by radiation to the extent that above a given mass, small postNewtonian corrections, negligible with regard to the hydrostatic structure, prevent the pressure from restoring the fragile equilibrium against adiabatic perturbations (Chandrasekhar 1964). For Pop III SMSs, the GR instability leads in general to the formation of a black hole seed of nearly the same mass as the progenitor (Fricke 1973; Shapiro \& Teukolsky 1979; Uchida et al. 2017), but once metals are included, thermonuclear explosions could prevent black hole formation (Appenzeller \& Fricke 1972a,b; Fuller et al. 1986; Montero et al. 2012). Such outcomes depend sensitively on the mass of the SMS at collapse, which is weakly constrained by the models (Woods et al. 2019), especially in the Pop I case.

In a previous work (Haemmerlé 2020), we showed how the onset point of the GR instability can be determined with a high level of accuracy from hydrostatic stellar models. However, the numerical estimates of the final masses suffer from the lack of such models up to the largest masses and accretion rates. The exploration of the parameter space with full stellar evolution is strongly limited by the numerical instability of stellar evolution codes when rapid accretion is included. In the present work, we circumvent this difficulty with the help of simplified analytical hydrostatic structures, built to match the structures obtained with full stellar evolution calculations accounting for rapid accretion. Hylotropes have been proposed by Begelman (2010) to describe the envelope of the "quasi-star" model, where 
a black hole grows at the centre of an SMS. Numerical models accounting for full stellar evolution showed that this class of structures describes with a high precision the actual envelope of SMSs in the extreme accretion regime (Haemmerlé et al. 2019). Here, we apply the method presented in Haemmerlé (2020) to the full class of hylotropes and discuss the implications regarding the final masses of SMSs in the various versions of direct collapse.

The main properties of rapidly accreting SMSs are reviewed in Sect. 2.1, with an emphasis on the physical motivations for the use of hylotropes. The methods we followed to build the hylotropic models and to determine their stability are described in Sects. 2.2 and 2.3, respectively. The results are presented in Sect. 3 and discussed in Sect. 4. We summarise our conclusions in Sect. 5.

\section{Method}

\subsection{The structure of rapidly accreting SMSs}

Relativistic corrections always remain small in SMSs. Considering their equilibrium properties, these stars are well-described by the classical equations of stellar structure (e.g. Hoyle \& Fowler 1963; Fuller et al. 1986; Hosokawa et al. 2013; Woods et al. 2017; Haemmerlé et al. 2018a). The equation of state is given by the sum of gas and radiation pressure:

$P=\frac{k_{\mathrm{B}}}{\mu m_{\mathrm{H}}} \rho T+\frac{1}{3} a_{\mathrm{SB}} T^{4}$,

where $P$ is the total pressure, $\rho$ the mass-density, $T$ the temperature, $\mu m_{\mathrm{H}}$ the average mass of the free particles of gas, $k_{\mathrm{B}}$ the Boltzmann constant, and $a_{\mathrm{SB}}$ is the Stefan-Boltzmann constant. They are always close to the Eddington limit, with an entropy $s_{\text {rad }}$ given by radiation and a ratio of gas to total pressure of the order of a percent:

$\beta:=\frac{P_{\mathrm{gas}}}{P}=\frac{1}{1+\frac{\mu m_{\mathrm{H}}}{3 k_{\mathrm{B}}} \frac{a_{\mathrm{SB}} T^{3}}{\rho}}=\frac{1}{1+\frac{\mu m_{\mathrm{H}}}{4 k_{\mathrm{B}}} s_{\mathrm{rad}}} \ll 1$.

Pressure can be expressed as a function of $\rho$ and $\beta$ :

$$
\begin{aligned}
P & =\left(\frac{3}{a_{\mathrm{SB}}}\right)^{1 / 3}\left(\frac{k_{\mathrm{B}}}{\mu m_{\mathrm{H}}}\right)^{4 / 3}\left(\frac{1-\beta}{\beta^{4}}\right)^{1 / 3} \rho^{4 / 3} \\
& \simeq\left(\frac{3}{a_{\mathrm{SB}}}\right)^{1 / 3}\left(\frac{k_{\mathrm{B}}}{\mu m_{\mathrm{H}}}\right)^{4 / 3} \beta^{-4 / 3} \rho^{4 / 3} .
\end{aligned}
$$

Due to convection driven by H-burning, the core of an SMS is isentropic $\left(\beta=\operatorname{cst}=: \beta_{c}\right)$, and therefore, according to Eq. (3), a polytrope of index $n=3$ :

$$
P=K \rho^{4 / 3}, \quad K=\left(\frac{3}{a_{\mathrm{SB}}}\right)^{1 / 3}\left(\frac{k_{\mathrm{B}}}{\mu m_{\mathrm{H}}}\right)^{4 / 3}\left(\frac{1-\beta}{\beta^{4}}\right)^{1 / 3}=\mathrm{cst}
$$

On the other hand, SMSs accrete at rates of $\dot{M}>0.1 M_{\odot} \mathrm{yr}^{-1}$, for which the accretion time $M / \dot{M}$ is shorter than the thermal timescale (Hosokawa et al. 2013; Haemmerlé et al. 2018a, 2019). As a consequence, the envelope is not thermally relaxed and maintains a higher entropy than the core, inhibiting convection. For rates of $\dot{M}>10 M_{\odot} \mathrm{yr}^{-1}$, thermal processes become completely negligible and the radiative envelope evolves adiabatically for most of its mass (Haemmerlé et al. 2019). In this case, the entropy profile builds homologously, which implies the following dependence on the mass coordinate, $M_{\mathrm{r}}(r$ is the radial coordinate):

$s \propto \frac{T^{3}}{\rho} \propto \frac{P^{3 / 4}}{\rho} \propto\left(\frac{M_{\mathrm{r}}^{2}}{r^{4}}\right)^{3 / 4} \frac{r^{3}}{M_{\mathrm{r}}}=M_{\mathrm{r}}^{1 / 2} \Longrightarrow \beta \propto M_{\mathrm{r}}^{-1 / 2}$,

where we used Eq. (2) to express $\beta$. With this scaling law, Eq. (3) gives a hylotrope (Begelman 2010):

$P=A M_{\mathrm{r}}^{2 / 3} \rho^{4 / 3}$,

where $A$ is a constant. The hylotropic law (6) switches into a polytropic law (4) at the limit of the convective core, that is, at a mass coordinate, $M_{\mathrm{r}}=M_{\text {core }}$. Continuity of pressure at the interface imposes

$K=A M_{\text {core }}^{2 / 3}$.

Thus, the structure of a maximally accreting SMS satisfies

$$
\begin{aligned}
& P=\left\{\begin{array}{ll}
K \rho^{4 / 3} & \text { if } M_{\mathrm{r}}<M_{\text {core }} \\
K\left(\frac{M_{\mathrm{r}}}{M_{\text {core }}}\right)^{2 / 3} \rho^{4 / 3} & \text { if } M_{\mathrm{r}}>M_{\text {core }}
\end{array},\right. \\
& \beta=\left\{\begin{array}{ll}
\beta_{c} & \text { if } M_{\mathrm{r}}<M_{\text {core }} \\
\beta_{c}\left(\frac{M_{\mathrm{r}}}{M_{\text {core }}}\right)^{-1 / 2} & \text { if } M_{\mathrm{r}}>M_{\text {core }}
\end{array},\right.
\end{aligned}
$$

with

$K=\left(\frac{3}{a_{\mathrm{SB}}}\right)^{1 / 3}\left(\frac{k_{\mathrm{B}}}{\mu m_{\mathrm{H}}}\right)^{4 / 3}\left(\frac{1-\beta_{c}}{\beta_{c}^{4}}\right)^{1 / 3}$.

The entropy profiles of the GENEC models of Haemmerlé et al. (2018a, 2019) at zero metallicity and $\dot{M}=1-1000 M_{\odot} \mathrm{yr}^{-1}$ are shown in Figs. 1-4. These profiles are similar in all respects to those found with other codes (Hosokawa et al. 2013; Umeda et al. 2016). The limit between the polytropic, convective core, and the hylotropic, radiative envelope appears clearly. An additional feature is visible, namely, the nearly vertical outer end of the profiles. The layers that are newly accreted gain entropy as they are incorporated in the stellar interior, which triggers convection. However, this impacts only the outer percent of the stellar mass, where the density is the lowest, and this feature can be neglected for our purpose. We see that for rates of $\dot{M} \geq 100 M_{\odot} \mathrm{yr}^{-1}$ the successive entropy profiles match each other in the envelope, following the hylotropic law (5). For $10 M_{\odot} \mathrm{yr}^{-1}$, the external layers depart slightly from the hylotropic law. For lower rates, the hylotropic approximation is broken in the whole envelope.

\subsection{Hylotropic structures}

Provided a constraint on pressure $P=f\left(\rho, M_{\mathrm{r}}\right)$, classical spherical stellar structures are fully determined by hydrostatic equilibrium and continuity of mass:

$$
\begin{aligned}
& \frac{\mathrm{d} P}{\mathrm{~d} r}=-\rho \frac{G M_{\mathrm{r}}}{r^{2}}, \\
& \frac{\mathrm{d} M_{r}}{\mathrm{~d} r}=4 \pi r^{2} \rho,
\end{aligned}
$$

where $G$ is the gravitational constant. We introduce the dimensionless functions $\xi, \theta, \varphi$ and $\psi$ :

$\xi=\alpha r, \quad \rho=\rho_{c} \theta^{3}, \quad \varphi=\frac{\alpha^{3}}{4 \pi \rho_{c}} M_{\mathrm{r}}, \quad P=P_{c} \psi$, 
L. Haemmerlé: General-relativistic instability in hylotropic supermassive stars

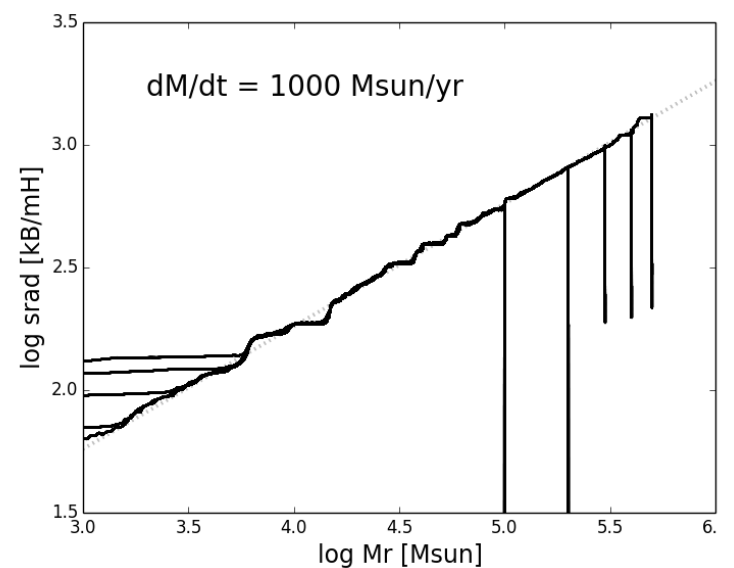

Fig. 1. Entropy profiles of the GENEC model at zero metallicity and $\dot{M}=1000 M_{\odot} \mathrm{yr}^{-1}$, at masses of $M=1-2-3-4-5 \times 10^{5} M_{\odot}$. The grey dotted line indicates the power-law $s=1.82 \frac{k_{\mathrm{B}}}{m_{\mathrm{H}}}\left(M_{\mathrm{r}} / M_{\odot}\right)^{1 / 2}$.

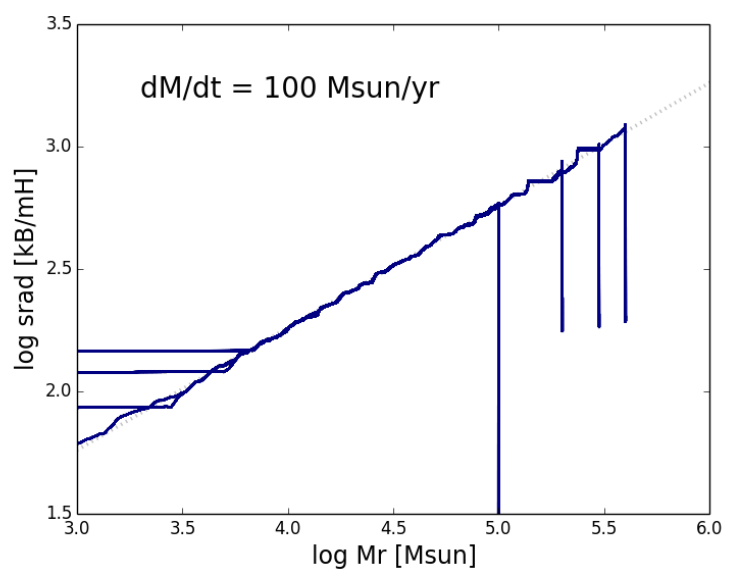

Fig. 2. Same as in Fig. 1 for $\dot{M}=100 M_{\odot} \mathrm{yr}^{-1}$ and $M=1-2-3-4 \times$ $10^{5} M_{\odot}$.

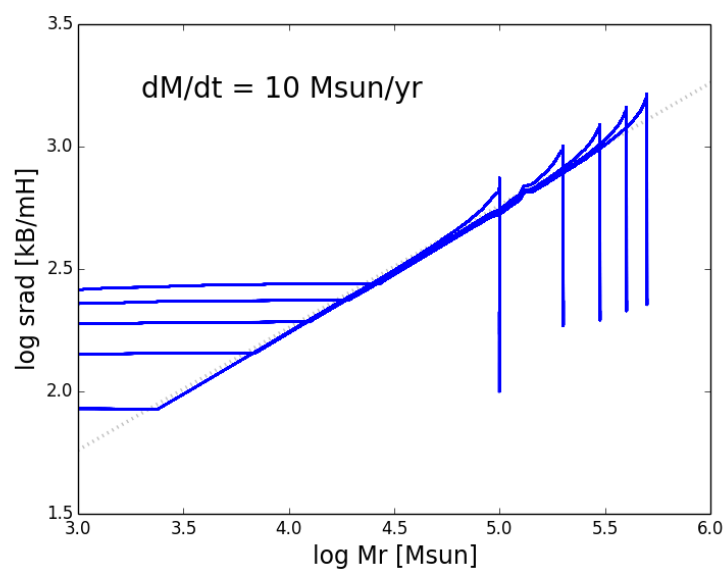

Fig. 3. Same as in Fig. 1 for $\dot{M}=10 M_{\odot} \mathrm{yr}^{-1}$ and $M=1-2-3-4-$ $5 \times 10^{5} M_{\odot}$.

where $\rho_{c}$ and $P_{c}$ are the central mass-density and pressure, and $\alpha$ is related to them by

$\alpha^{2}=\frac{\pi G \rho_{c}^{2}}{P_{c}}$.

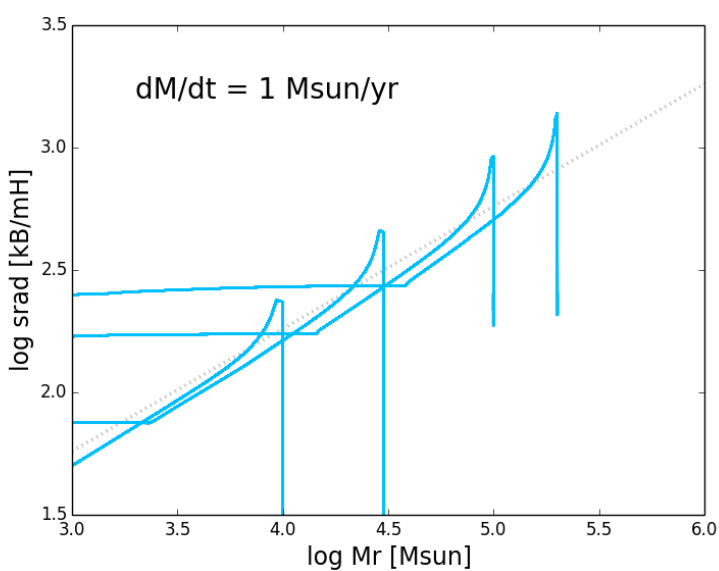

Fig. 4. Same as in Fig. 1 for $\dot{M}=1 M_{\odot} \mathrm{yr}^{-1}$ and $M=0.1-0.3-1-2 \times$ $10^{5} M_{\odot}$.

The boundary conditions are given by

$r=0: \xi=0, \theta=\psi=1, \varphi=0$,

$r=R \quad: \quad \xi=\alpha R=: \xi_{\text {surf }}, \quad \theta=\psi=0, \quad \varphi=\frac{\alpha^{3} M}{4 \pi \rho_{c}}=: \varphi_{\text {surf }}$,

where $M$ and $R$ are the total mass and radius of the star, with $\varphi_{\text {surf }}$ and $\xi_{\text {surf }}$ as their dimensionless equivalents. With these dimensionless functions, Eqs. (11)-(12) become

$$
\begin{aligned}
& \frac{\mathrm{d} \psi}{\mathrm{d} \xi}=-4 \frac{\varphi \theta^{3}}{\xi^{2}}, \\
& \frac{\mathrm{d} \varphi}{\mathrm{d} \xi}=\xi^{2} \theta^{3} .
\end{aligned}
$$

The constraint on pressure reduces to a function $\psi(\theta, \varphi)$ that closes Eqs. (17)-(18). Translated into the dimensionless functions (13), Eqs. (8)-(9) give

$\psi=\left\{\begin{array}{ll}\theta^{4} & \text { if } \varphi<\varphi_{\text {core }} \\ \left(\frac{\varphi}{\varphi_{\text {core }}}\right)^{2 / 3} \theta^{4} & \text { if } \varphi>\varphi_{\text {core }}\end{array}\right.$,

$\beta=\left\{\begin{array}{ll}\beta_{c} & \text { if } \varphi<\varphi_{\text {core }} \\ \beta_{c}\left(\frac{\varphi}{\varphi_{\text {core }}}\right)^{-1 / 2} & \text { if } \varphi>\varphi_{\text {core }}\end{array}\right.$,

where

$\varphi_{\text {core }}:=\frac{\alpha^{3}}{4 \pi \rho_{c}} M_{\text {core }}$

is the dimensionless mass of the convective core which remains the only free parameter in the equation system.

Hylotropic structures can be built by numerical integration of (17)-(18) with constraint (19), starting from central conditions (15). For the numerical integration of the hylotropic envelope $\left(\varphi>\varphi_{\text {core }}\right)$, we follow the more suitable Lagrangian formulation of Begelman (2010) who defines $w=\mathrm{d} \ln \xi / \mathrm{d} \ln \varphi$. In terms of this variable, Eqs. (11)-(12) with (6) lead to

$$
\begin{aligned}
\frac{\mathrm{d} \ln \varphi}{\mathrm{d} \ln w} & =\frac{1}{\frac{3}{2}-3 w+3 \varphi_{\mathrm{core}}^{2 / 3} w^{4 / 3}}, \\
\frac{\mathrm{d} \ln \xi}{\mathrm{d} \ln w} & =\frac{w}{\frac{3}{2}-3 w+3 \varphi_{\mathrm{core}}^{2 / 3} w^{4 / 3}} .
\end{aligned}
$$


The functions $\theta, \psi$ and $\beta$ are then obtained by Eqs. (18)-(20). Since $w \rightarrow \infty$ when $\rho \rightarrow 0$, integration with respect to $w$ never reaches the surface. We stop the integration when $\theta<10^{-8}$. We build in this way a series of hylotropes with $\varphi_{\text {core }}$ ranging in the interval:

$\varphi_{\min }:=0.459<\varphi_{\text {core }}<2.02=: \varphi_{\max }$

by steps of $0.05-0.3$. The lower limit of this interval is imposed by the condition of gravitational binding (Begelman 2010), while the upper limit corresponds to polytropes, that is, $\varphi_{\text {core }}=$ $\varphi_{\text {surf }}$.

The dimensional quantities can then be derived from the dimensionless ones. The polytropic law (4) in the core implies $P_{c}=K \rho_{c}^{4 / 3}$. With the definition (14) of $\alpha$ and the expression (10) of $K$, it gives a mass scale of

$\frac{4 \pi \rho_{c}}{\alpha^{3}}=\frac{4}{\sqrt{\pi}}\left(\frac{K}{G}\right)^{3 / 2}=\frac{4}{G^{3 / 2}}\left(\frac{3}{\pi a_{\mathrm{SB}}}\right)^{1 / 2}\left(\frac{k_{\mathrm{B}}}{\mu m_{\mathrm{H}}}\right)^{2}\left(\frac{1-\beta_{c}}{\beta_{c}^{4}}\right)^{1 / 2}$.

We see that the mass scale is fully determined by $\beta_{c}$ and the chemical composition. The choice of the central temperature, imposed by H-burning, determines $\rho_{c}$ via Eq. (2), which defines the length-scale $\alpha^{-1}$ through the mass scale (25).

\subsection{GR instability in the post-Newtonian + Eddington limit}

We showed in Haemmerlé (2020) that the onset point of the GR instability can be determined with high accuracy from numerical hydrostatic structures by a simple application of the relativisitic equation of adiabatic pulsation of Chandrasekhar (1964) in the following form:

$\frac{\omega^{2}}{c^{2}} I_{0}=\sum_{i=1}^{4} I_{i}$

with

$I_{0}=\int_{0}^{R} e^{a+3 b}\left(P+\rho c^{2}\right) r^{4} \mathrm{~d} r$,

$I_{1}=9 \int_{0}^{R} e^{3 a+b}\left(\Gamma_{1}-\frac{4}{3}\right) \operatorname{Pr}^{2} \mathrm{~d} r$

$I_{2}=-12 \int_{0}^{R} e^{3 a+b}\left(a^{\prime}+\frac{b^{\prime}}{3}\right) \operatorname{Pr}^{3} \mathrm{~d} r$,

$I_{3}=\frac{8 \pi G}{c^{4}} \int_{0}^{R} e^{3(a+b)} P\left(P+\rho c^{2}\right) r^{4} \mathrm{~d} r$

$I_{4}=-\int_{0}^{R} e^{3 a+b} \frac{P^{\prime 2}}{P+\rho c^{2}} r^{4} \mathrm{~d} r$,

where' indicates the derivatives with respect to the radial coordinate $r$ ( $r=R$ at the surface), $P$ is the pressure, $\rho$ the density of relativistic mass, $c$ the speed of light, $G$ the gravitational constant, $\Gamma_{1}$ the first adiabatic exponent, and $a$ and $b$ are the coefficients of the metric:

$\mathrm{d} s^{2}=-e^{2 a}(c \mathrm{~d} t)^{2}+e^{2 b} \mathrm{~d} r^{2}+r^{2} \mathrm{~d} \Omega^{2}$.

Einstein's equations lead to the following expressions for a spherical, static metric:

$a^{\prime}=\frac{G M_{\mathrm{r}}}{r^{2} c^{2}} \frac{1+\frac{4 \pi r^{3}}{M_{\mathrm{r}} c^{2}} P}{1-\frac{2 G M_{\mathrm{r}}}{r c^{2}}}, \quad e^{2 a(R)}=1-\frac{2 G M}{R c^{2}}$,

$e^{-2 b}=1-\frac{2 G M_{\mathrm{r}}}{r c^{2}}$.
The relativistic mass-coordinates $M_{\mathrm{r}}\left(M=M_{R}\right)$ is related to the other quantities by Eq. (12) with relativistic-mass instead of restmass in $M_{\mathrm{r}}$ and $\rho$.

The condition for GR instability is

$$
\sum_{i=1}^{4} I_{i}<0
$$

Here, we formulate this condition for the special case of postNewtonian stars near the Eddington limit. To that aim, we reexpress integrals $I_{i}$ in the dimensionless functions (13). Following Tooper (1964a), we also define:

$\sigma:=\frac{P_{c}}{\rho_{c} c^{2}}=\frac{\pi G \rho_{c}}{\alpha^{2} c^{2}}$

which represents the dimensionless parameter for the relativistic correction since compactness is given by

$\frac{2 G M_{\mathrm{r}}}{r c^{2}}=8 \sigma \frac{\varphi}{\xi}$.

In these dimensionless quantities, integrals (28)-(31) are given as:

$I_{1}=9 \frac{P_{c}}{\alpha^{3}} \int e^{3 a+b}\left(\Gamma_{1}-\frac{4}{3}\right) \psi \xi^{2} \mathrm{~d} \xi$,

$I_{2}=-12 \frac{P_{c}}{\alpha^{3}} \int e^{3 a+b}\left(\frac{\mathrm{d} a}{\mathrm{~d} \xi}+\frac{1}{3} \frac{\mathrm{d} b}{\mathrm{~d} \xi}\right) \psi \xi^{3} \mathrm{~d} \xi$,

$I_{3}=8 \sigma \frac{P_{c}}{\alpha^{3}} \int e^{3(a+b)}\left(1+\sigma \frac{\psi}{\theta^{3}}\right) \psi \theta^{3} \xi^{4} \mathrm{~d} \xi$,

$I_{4}=-\sigma \frac{P_{c}}{\alpha^{3}} \int e^{3 a+b} \frac{\left(\frac{\mathrm{d} \psi}{\mathrm{d} \xi}\right)^{2}}{1+\sigma \frac{\psi}{\theta^{3}}} \frac{\xi^{4} \mathrm{~d} \xi}{\theta^{3}}$,

where the integration is made over the interval $0<\xi<\xi_{\text {surf }}$. Each integral has the same dimensional factor $P_{c} / \alpha^{3}$ in front, which can be extracted from the sum and cancelled via the inequality (35).

In the Eddington limit, the ratio of gas to total pressure is $\beta \ll 1$ and the first adiabatic exponent can be evaluated as

$\Gamma_{1}=\frac{4}{3}+\frac{\beta}{6}=\frac{4}{3}+\frac{\beta_{\mathrm{c}}}{6} \frac{\beta}{\beta_{c}}$.

We take the central value $\beta_{c} \ll 1$ as the parameter for the departures from the Eddington limit, such as $\sigma \ll 1$ represents the departures from the Newtonian limit. For post-Newtonian stars near the Eddington limit, integrals (38)-(41)) can be developed in these two parameters, neglecting second-order terms, $O\left(\sigma^{2}\right)$ or $O\left(\beta_{c} \sigma\right)$. We notice that with the previously used Eq. (42), each integral has a global $\sigma$ or $\beta_{c}$ factor, except for $I_{2}$, where the $\sigma$ factor has to be extracted from the derivatives of the metric. These derivatives are taken from Eqs. (33)-(34) and we only need the $O(\sigma)$ terms:

$\frac{\mathrm{d} a}{\mathrm{~d} \xi}=4 \sigma \frac{\varphi}{\xi^{2}}$,

$\frac{\mathrm{d} b}{\mathrm{~d} \xi}=-4 \sigma \frac{\varphi}{\xi^{2}}+4 \sigma \xi \theta^{3}$,

(using Eq. (18)). In this way, each integral has either a global $\sigma$ or $\beta_{c}$ factor, which makes all the other relativistic corrections useless. In particular, we can ignore all other departures from Minkowskian metric and take $e^{3 a+b}=e^{3(a+b)}=1$. Finally, the 
pressure gradient in $I_{4}$ can be eliminated with classical hydrostatic equilibrium (17). Once we remove all the second-order terms, the integrals read:

$I_{1}=\frac{3}{2} \beta_{c} \frac{P_{c}}{\alpha^{3}} \int \frac{\beta}{\beta_{c}} \psi \xi^{2} \mathrm{~d} \xi$,

$I_{2}=-16 \sigma \frac{P_{c}}{\alpha^{3}} \int\left(\xi \theta^{3}+\frac{2 \varphi}{\xi^{2}}\right) \psi \xi^{3} \mathrm{~d} \xi$

$I_{3}=8 \sigma \frac{P_{c}}{\alpha^{3}} \int \psi \theta^{3} \xi^{4} \mathrm{~d} \xi$,

$I_{4}=-16 \sigma \frac{P_{c}}{\alpha^{3}} \int \varphi^{2} \theta^{3} \mathrm{~d} \xi$,

and their sum is

$$
\begin{aligned}
\frac{2 \alpha^{3}}{3 P_{c}} \sum_{i=1}^{4} I_{i}=\beta_{c} & \int \frac{\beta}{\beta_{c}} \psi \xi^{2} \mathrm{~d} \xi \\
& -\frac{16}{3} \sigma\left(\int \xi^{4} \psi \theta^{3} \mathrm{~d} \xi+2 \int \varphi^{2} \theta^{3} \mathrm{~d} \xi\right. \\
& \left.+4 \int \xi \psi \varphi \mathrm{d} \xi\right) .
\end{aligned}
$$

The first integral in the $\sigma$ term can be expressed as a function of the other two, using (i) the continuity Eq. (18), (ii) an integration by parts (boundary terms vanish), (iii) hydrostatic equilibrium (17):

$$
\begin{array}{r}
\int \xi^{4} \psi \theta^{3} \mathrm{~d} \xi \stackrel{(\mathrm{i})}{=} \int \xi^{2} \psi \frac{\mathrm{d} \varphi}{\mathrm{d} \xi} \mathrm{d} \xi \stackrel{(\mathrm{ii})}{=}-\int \varphi \frac{\mathrm{d}}{\mathrm{d} \xi}\left(\xi^{2} \psi\right) \mathrm{d} \xi \\
\stackrel{(\text { iii) }}{=}-2 \int \xi \psi \varphi \mathrm{d} \xi+4 \int \varphi^{2} \theta^{3} \mathrm{~d} \xi
\end{array}
$$

As a consequence, the GR instability occurs when

$$
\frac{\beta_{c}}{\sigma}<\frac{32}{3} \frac{\int \xi \psi \varphi \mathrm{d} \xi+3 \int \varphi^{2} \theta^{3} \mathrm{~d} \xi}{\int \frac{\beta}{\beta_{c}} \psi \xi^{2} \mathrm{~d} \xi}=:\left.\frac{\beta}{\sigma}\right|_{\text {crit }} .
$$

Since we extracted already the post-Newtonian correction through $\sigma$, the integrals in Eq. (54) can be evaluated numerically from Newtonian structures $(\sigma \rightarrow 0)$, either numerical or analytical. Departures from the Eddington limit are already included in the factor $\beta_{c}$ and the function $\beta / \beta_{c}$, that is, by the effect of gas pressure and we do not need to account for corrections due to the entropy of gas, which justifies a choice of the index, namely, $n=3$, in the core (Sect. 2.1). Thus, it allows us to consistently capture the GR instability on the classical hylotropic models displayed in Sect. 2.2. Distinctions between relativistic- and restmass in the hydrostatic structures are insignficant.

For given dimensionless structures, determined by the functions $\theta(\xi), \varphi(\xi), \psi(\xi)$, and $\beta(\xi) / \beta_{c}$, Eq. (54) gives a critical ratio of $\beta_{c}$ and $\sigma$. On the other hand, by definition of $\beta_{c}(1)-(2)$ and $\sigma$ (36), the actual product of these two quantities is directly given by the central temperature, $T_{\mathrm{c}}$, and the chemical composition:

$\beta_{c} \sigma=\frac{k_{\mathrm{B}} T_{\mathrm{c}}}{\mu m_{\mathrm{H}} c^{2}}$.

Thus, for given dimensionless structures, the properties of the star at the limit of stability are fully determined by central temperature and chemical composition, since $\beta_{c}$ and $\sigma$ are both defined.

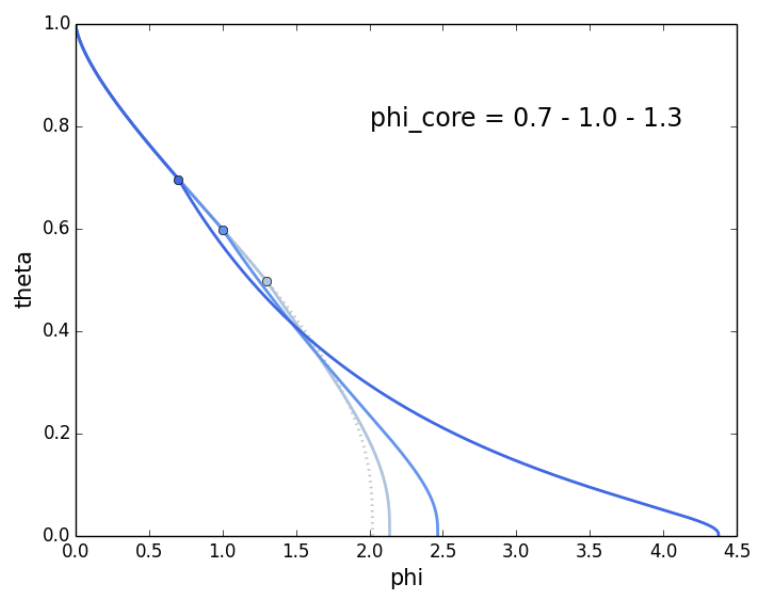

Fig. 5. Hylotropic structures built according to Sect. 2.2, for the indicated values of $\varphi_{\text {core }}$, the dimensionless mass of the core. The dotted line is the polytrope used for the core and a circle indicates $\varphi_{\text {core }}$ for the various models.

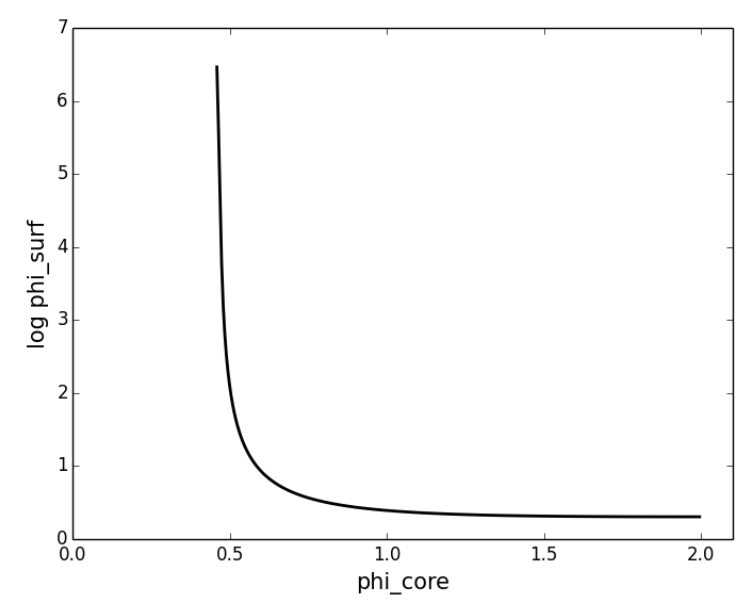

Fig. 6. Total dimensionless mass as a function of the dimensionless mass of the core for the series of hylotropic structures built according to Sect. 2.2.

\section{Results}

From the full series of hylotropic structures built according to Sect. (2.2), three of them are shown in Fig. 5, together with the polytrope. As noted by Begelman (2010), a decrease in $\varphi_{\text {core }}$ translates into an increase in $\varphi_{\text {surf }}$, the total dimensionless mass. This is because the pressure gradient is flatter with the hylotropic law than with the polytropic one due to the dependence on the mass coordinate. Thus, larger masses are reached before pressure and density vanish. To show this effect more clearly, we plot $\varphi_{\text {surf }}$ as a function of $\varphi_{\text {core }}$ for the full series of structures in Fig. 6 (we note the logarithmic scale in the $y$-axis). The increase remains moderate down to $\varphi_{\text {core }} \gtrsim 1$, but then $\varphi_{\text {surf }}$ gains orders of magnitude to reach millions when $\varphi_{\text {core }} \rightarrow \varphi_{\text {min }}$. The mass fraction of the core as a function of $\xi_{\text {core }}$ in Begelman (2010) is well-reproduced by our series of structures.

For this series of structures, we inject the functions $\theta(\xi), \varphi(\xi)$, $\psi(\xi)$, and $\beta(\xi) / \beta_{c}$ from Eqs. 19-(20) into the stability condition (54). It gives the critical ratio $\left.\frac{\beta}{\sigma}\right|_{\text {crit }}$ as a function of $\varphi_{\text {core }}$, which is shown in Fig. 7. In the polytropic limit $\varphi_{\text {core }} \rightarrow \varphi_{\max }$, we find a value 15.8 that reproduces the numerical tables of Chandrasekhar (1964) and Tooper (1964b) well. When $\varphi_{\text {core }}<$ $\varphi_{\text {surf }}$, the critical ratio departs towards larger values. It could be 


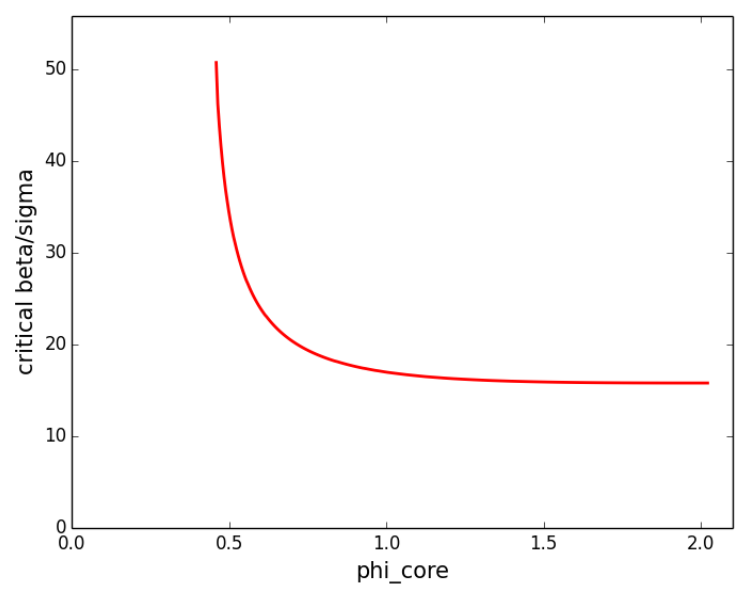

Fig. 7. Critical ratio $\left.\frac{\beta}{\sigma}\right|_{\text {crit }}$ of Eq. (54) for the series of hylotropic structures built according to the steps detailed in Sect. 2.2.

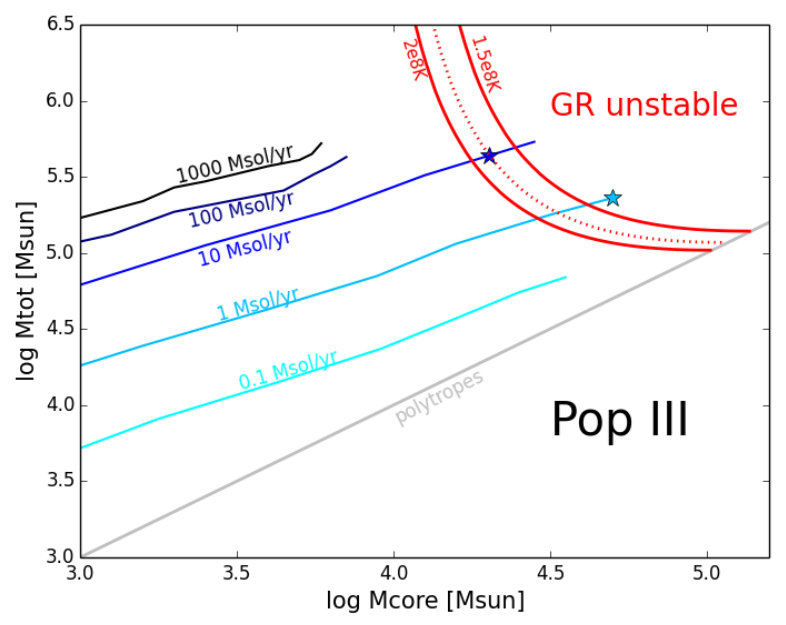

Fig. 8. Total mass of the star versus mass of the convective core in the Pop III case. Solid red lines indicate the limit of stability for hylotropic models with $\mu=0.6$ and $T_{c}=1.5-2 \times 10^{8} \mathrm{~K}$. The GENEC models of Haemmerlé et al. (2018a, 2019) at zero metallicity are plotted with the indicated rates. The identity corresponds to polytropes of different masses, and is shown as a grey line. The final masses obtained in Haemmerlé (2020) are marked by star-like symbols. The dotted red line is the limit of stability for hylotropes with $\mu=0.6$ and the exact central temperature of the GENEC model at $10 M_{\odot} \mathrm{yr}^{-1}$ at the onset of instability $\left(T_{c}=1.78 \times 10^{8} \mathrm{~K}\right)$.

interpreted naively as a destabilising effect, but it reflects only the increase in $\varphi_{\text {surf }}$ that results from the decrease in $\varphi_{\text {core }}$. In other words, we compare objects of different masses. We can see already that when the total dimensionless mass gains several orders of magnitude (Fig. 6), the critical ratio $\left.\frac{\beta}{\sigma}\right|_{\text {crit }}$ grows only by a factor of a few, which suggests that hylotropes with low $\varphi_{\text {core }}$ remain stable up to larger masses than polytropes.

In order to quantify this effect, we fix the product, $\beta_{c} \sigma$, according to Eq. (55), and impose critical conditions $\beta_{c} / \sigma=$ $\left.\frac{\beta}{\sigma}\right|_{\text {crit }}$. The central temperature of Pop III SMSs is always comprised in the interval $1.5-2 \times 10^{8} \mathrm{~K}$ and the chemical composition does not depart significantly from $\mu=0.6$ (Hosokawa et al. 2013; Haemmerlé et al. 2018a). With these values in Eq. (55) and the critical condition, $\beta_{c}$, is determined, which gives the mass-scale via Eq. (25). This allows us to express the mass of the star and that of the core in solar units.

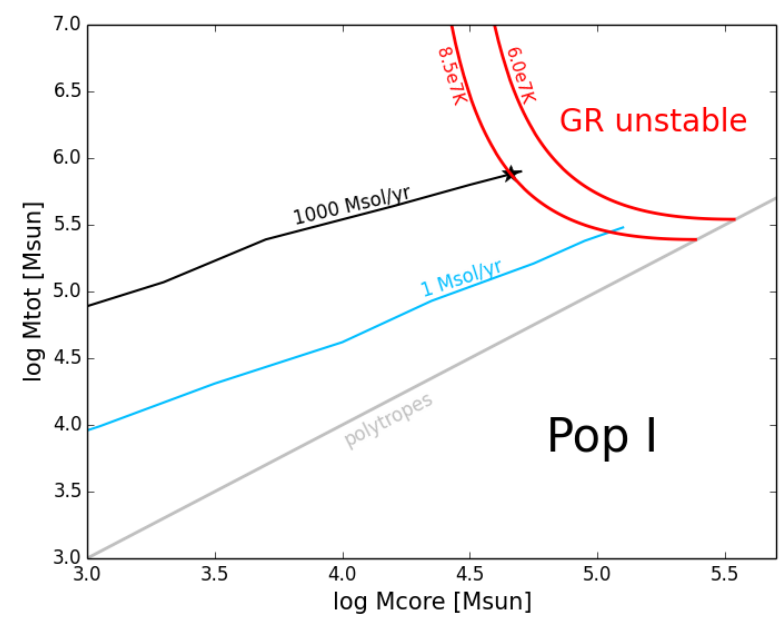

Fig. 9. Same as in Fig. 8 in the Pop I case.

The result of this treatment is shown in Fig. 8. We see that the changes in central temperature over the narrow range of relevant values does not change the stability limit significantly. For polytropic structures, the maximum mass consistant with stability is $1-1.5 \times 10^{5} M_{\odot}$, which is in agreement with Woods et al. (2020). However, when the mass fraction of the convective core decreases, we see that the limit of stability moves towards larger masses. In other words, the hylotropic law in the envelope has a stabilising effect against adiabatic pulsations as it decreases the compactness. The effect becomes extremely strong when the mass of the core stays as low as $\sim 10^{4} M_{\odot}$. In this case, masses in excess of $10^{6} M_{\odot}$ could be reached, resulting from the sensitive dependence of the total mass on the core mass when $\varphi_{\text {core }}<1$, while the critical ratio $\left.\frac{\beta}{\sigma}\right|_{\text {crit }}$ does not change much, as noted above. Thus, the mass of the convective core appears as a key quantity and a star hardly reaches instability if its convective core does not exceed $\sim 10^{4} M_{\odot}$. It is only for total masses of $\gtrsim 10^{7} M_{\odot}$ (not shown in the figure) that the stability limit moves to $M_{\text {core }}<10^{4} M_{\odot}$. At the limit of gravitational binding $\left(\varphi_{\text {core }} \rightarrow \varphi_{\text {min }}\right.$, Eq. (24)), we obtain the largest mass consistant with stable equilibrium, which is $\sim 5 \times 10^{10} M_{\odot}$, with a core of $\sim 5000 M_{\odot}$ (i.e. a core mass fraction of $10^{-7}$ ).

In the Pop I case, shown in Fig. 9, the lower central temperatures shift the stability limit towards higher masses, allowing it to reach $\sim 3-5 \times 10^{5} M_{\odot}$ in the polytropic limit. At the same time, the typical core's mass required for GR instability moves up to $\sim 3-5 \times 10^{4} M_{\odot}$.

\section{Discussion}

The reliability of the hylotropic models can be tested by a comparison with the results of the GENEC models (Haemmerlé et al. 2018a, 2019; Haemmerlé 2020). The entropy profiles of these models suggest that hylotropes are relevant for rates of $\gtrsim 100 M_{\odot} \mathrm{yr}^{-1}$ (Sect. 2.1). The GENEC tracks are displayed in Fig. 8 for zero metallicity, with their collapse points indicated as star-like symbols. The comparison can be made only for 1 and $10 M_{\odot} \mathrm{yr}^{-1}$ since the other models are still a substantial distance from any instability. We see that the last stable model at $10 M_{\odot} \mathrm{yr}^{-1}$ lies in the critical band of hylotropic models with $T_{c}=1.5-2 \times 10^{8} \mathrm{~K}$, while the one at $1 M_{\odot} \mathrm{yr}^{-1}$ goes slightly over the limit. To refine the comparison, we plot as a red dotted line the hylotropic limit with the exact central temperature of the GENEC model at $10 M_{\odot} \mathrm{yr}^{-1}$ at the onset point of instability $\left(T_{c}=1.78 \times 10^{8} \mathrm{~K}\right)$. We see that the matching is 
perfect. Departures from hylotropy in the entropy profile of this model (Fig. 3) occur only near the surface, where the density is low, which implies negligible changes in the GR corrections. It is only for rates of $\leq 1 M_{\odot} \mathrm{yr}^{-1}$ that the entropy profiles depart from hylotropy in the inner envelope (Fig. 4). We note that the central temperature of the last stable model at $1 M_{\odot} \mathrm{yr}^{-1}$ is very close to $1.5 \times 10^{8} \mathrm{~K}$, so that the upper red curve gives the hylotropic limit relevant for this model. Thus, even at this rate, the hylotropic limit is only exceeded by $\sim 0.1$ dex in the $\left(M, M_{\text {core }}\right)$ diagram. An extrapolation of the curve at $0.1 M_{\odot} \mathrm{yr}^{-1}$ suggests that this model, if it ever reaches the GR instability during H-burning, does so as a fully relaxed, polytropic star. Overall, it appears difficult to find a rate for which the hylotropic limit would be significantly exceeded. For rates of $>1000 M_{\odot} \mathrm{yr}^{-1}$, an extrapolation of the tracks suggests final masses in excess of $10^{6} M_{\odot}$.

The GENEC tracks at solar metallicity are plotted in Fig. 9 for the two available rates, namely, 1 and $1000 M_{\odot} \mathrm{yr}^{-1}$. The central temperatures that have been used for the hylotropic models correspond to that of the last stable structure of the $1000 M_{\odot} \mathrm{yr}^{-1}$ run $\left(8.5 \times 10^{7} \mathrm{~K}\right)$ and of the last model of the $1 M_{\odot} \mathrm{yr}^{-1}$ run $\left(6 \times 10^{7} \mathrm{~K}\right)$, which is still far from instability. We see that the larger convective core of Pop I models cancels the effect of the lower central temperature, as the tracks are shifted with the stability limit. As expected, the stability limit given by the hylotropic models with the relevant central temperature crosses the $1000 M_{\odot} \mathrm{yr}^{-1}$ track at the exact point of instability obtained in Haemmerlé (2020), which corresponds to a mass of $759000 M_{\odot}$. The comparison cannot be done for lower rates due to the lack of models. As in the Pop III case, masses of $>10^{6} M_{\odot}$ require rates of $>1000 M_{\odot} \mathrm{yr}^{-1}$.

These results confirm that spherical SMSs forming in atomically cooled haloes cannot reach masses in excess of $500000 M_{\odot}$, while masses in excess of $10^{6} M_{\odot}$ could be reached in the galaxy merger scenario (see Sect. 1), provided accretion rates of $\gtrsim 1000 M_{\odot} \mathrm{yr}^{-1}$. In fact, such rates appear necessary for massive black hole formation through Pop I galaxy mergers since at solar metallicity, masses of $\gtrsim 10^{6} M_{\odot}$ are required to avoid thermonuclear explosion during the collapse (Montero et al. 2012). In this respect, the final mass of the black hole progenitor ranges across distinct intervals throughout the various versions of direct collapse: $\$ 500000 M_{\odot}$ for atomically cooled haloes; this value is $\gtrsim 10^{6} M_{\odot}$ for Pop I galaxy mergers.

We note that this picture could change significantly when including rotation. Given enough angular momentum, the centrifugal force has been shown to remove the GR instability in polytropic models up to masses of $\sim 10^{8} M_{\odot}$ (Fowler 1966; Bisnovatyi-Kogan et al. 1967). On the other hand, rapidly accreting SMSs must be slow rotators (Haemmerlé et al. 2018b) due to the $\Omega \Gamma$-limit (Maeder \& Meynet 2000). For masses of $>10^{5} M_{\odot}$, their surface velocity cannot exceed a few percents of the Keplerian velocity. The impact of rotation on the stability of hylotropic models shall be addressed in a forthcoming work.

\section{Summary and conclusions}

In this work, we estimate the final masses of the progenitors of massive black hole seeds in the various versions of direct collapse on the basis of hylotropic models built according to Begelman (2010). Through a comparison with the GENEC models, accounting for full stellar evolution (Haemmerlé et al. 2018a, 2019; Haemmerlé 2020), we show that hylotropic models are able to predict the final masses with a high level of accuracy for accretion rates of $\geq 10 M_{\odot} \mathrm{yr}^{-1}$ and it remains a very good approximation even for lower rates.
The hylotropic law in the envelope has a stabilising effect against adiabatic perturbations to equilibrium as it decreases the compactness and, thus, reduces the destabilising GR effects. The mass of the convective core is consequential for the stability of the star. The lower the core's mass, the larger the total mass consistent with stable equilibrium (Figs. 8-9). Typical conditions for GR instability are a total mass $\gtrsim 10^{5} M_{\odot}$ with a core mass $\gtrsim 10^{4} M_{\odot}$. For a core mass as low as $\lesssim 10^{4} M_{\odot}$, total masses in excess of $10^{6}-10^{7} M_{\odot}$ remain consistent with stability.

These results confirm that spherical SMSs forming in atomically cooled haloes (Pop III, $\dot{M} \lesssim 10 M_{\odot} \mathrm{yr}^{-1}$ ) have masses of $\$ 500000 M_{\odot}$. With regard to SMSs formed by accretion at rates of $\gtrsim 1000 M_{\odot} \mathrm{yr}^{-1}$, these could reach masses of $\gtrsim 10^{6} M_{\odot}$, which is a required condition for massive black hole formation in Pop I galaxy mergers. It implies that the final masses of the progenitors of massive black hole seeds range across distinct intervals throughout the various versions of direct collapse.

Acknowledgements. LH has received funding from the European Research Council (ERC) under the European Union's Horizon 2020 research and innovation programme (grant agreement No 833925, project STAREX).

\section{References}

Appenzeller, I., \& Fricke, K. 1972a, A\&A, 18, 10 Appenzeller, I., \& Fricke, K. 1972b, A\&A, 21, 285 Begelman, M. C. 2010, MNRAS, 402, 673

Bisnovatyi-Kogan, G. S., Zel'dovich, Y. B., \& Novikov, I. D. 1967, Sov. Ast., 11, 419

Bromm, V., \& Loeb, A. 2003, ApJ, 596, 34

Chandrasekhar, S. 1964, ApJ, 140, 417

Dijkstra, M., Haiman, Z., Mesinger, A., \& Wyithe, J. S. B. 2008, MNRAS, 391, 1961

Fowler, W. A. 1966, ApJ, 144, 180

Fricke, K. J. 1973, ApJ, 183, 941

Fuller, G. M., Woosley, S. E., \& Weaver, T. A. 1986, ApJ, 307, 675

Haemmerlé, L. 2020, A\&A, submitted [arXiv:2010. 08229]

Haemmerlé, L., \& Meynet, G. 2019, A\&A, 623, L7

Haemmerlé, L., Woods, T. E., Klessen, R. S., Heger, A., \& Whalen, D. J. 2018a, MNRAS, 474, 2757

Haemmerlé, L., Woods, T. E., Klessen, R. S., Heger, A., \& Whalen, D. J. 2018b, ApJ, 853, L3

Haemmerlé, L., Meynet, G., Mayer, L., et al. 2019, A\&A, 632, L2

Haemmerlé, L., Mayer, L., Klessen, R. S., et al. 2020, Space Sci. Rev., 216, 48 Haiman, Z., Rees, M. J., \& Loeb, A. 1997, ApJ, 476, 458

Hosokawa, T., Yorke, H. W., Inayoshi, K., Omukai, K., \& Yoshida, N. 2013, ApJ, 778,178

Hoyle, F., \& Fowler, W. A. 1963, MNRAS, 125, 169

Latif, M. A., Schleicher, D. R. G., Schmidt, W., \& Niemeyer, J. C. 2013, MNRAS, 436, 2989

Maeder, A., \& Meynet, G. 2000, A\&A, 361, 159

Mayer, L., \& Bonoli, S. 2019, Rep. Prog. Phys., 82, 016901

Mayer, L., Kazantzidis, S., Escala, A., \& Callegari, S. 2010, Nature, 466, 1082

Mayer, L., Fiacconi, D., Bonoli, S., et al. 2015, ApJ, 810, 51

Montero, P. J., Janka, H.-T., \& Müller, E. 2012, ApJ, 749, 37

Omukai, K. 2001, ApJ, 546, 635

Rees, M. J. 1978, The Observatory, 98, 210

Rees, M. J. 1984, ARA\&A, 22, 471

Sakurai, Y., Hosokawa, T., Yoshida, N., \& Yorke, H. W. 2015, MNRAS, 452, 755

Schleicher, D. R. G., Palla, F., Ferrara, A., Galli, D., \& Latif, M. 2013, A\&A, 558, A59

Shapiro, S. L., \& Teukolsky, S. A. 1979, ApJ, 234, L177

Tooper, R. F. 1964a, ApJ, 140, 434

Tooper, R. F. 1964b, ApJ, 140, 811

Uchida, H., Shibata, M., Yoshida, T., Sekiguchi, Y., \& Umeda, H. 2017, Phys. Rev. D, 96, 083016

Umeda, H., Hosokawa, T., Omukai, K., \& Yoshida, N. 2016, ApJ, 830, L34

Volonteri, M., Lodato, G., \& Natarajan, P. 2008, MNRAS, 383, 1079

Woods, T. E., Heger, A., Whalen, D. J., Haemmerlé, L., \& Klessen, R. S. 2017, ApJ, 842, L6

Woods, T. E., Agarwal, B., Bromm, V., et al. 2019, PASA, 36, e027

Woods, T. E., Heger, A., \& Haemmerlé, L. 2020, MNRAS, 494, 2236 